\title{
Projet de stockage intersaisonnier de calories climatiques en aquifère
}

\section{Project for Interseasonal Storage of Climatic Calories in an Aquifer}

E. Landon

BURGEAP

\author{
J.P. Petit
}

70, rue Mademoiselle, 75015 Paris

\begin{abstract}
Les auteurs présentent les modifications qui ont été apportées au projet d'un système de chauffage solaire associé à un stockage en aquifère, et qui avait été terminé en 1982, compte-tenu de l'évolution ultérieure du contexte énergétique et économique.
\end{abstract}

The authors describe the modifications made to the project for a system of solar heating associated with storage in aquifer, which was completed in 1982, to take into account later developments in the energy and economic context.

\section{Introduction}

Le stockage d'eau chaude dans les aquifères, déjà étudié à plusieurs reprises, est un procédé intéressant lorsque les conditions hydrogéologiques du site sont favorables. Il permet de mettre en valeur des sources d'énergie diverses comme par exemple les rejets thermiques industriels ou l'énergie solaire.

L'étude de faisabilité et le projet dont il est question aujourd'hui ont été lancés au temps du développement du solaire dit "actif ". L'objectif était de montrer l'intérêt compétitif d'un système solaire, dimensionné en fonction des apports d'été, associé à un stockage en aquifère pour le chauffage d'un groupe scolaire situé dans la Ville Nouvelle de St-Quentin-en-Yvelines. L'étude concluait sur l'intérêt de réaliser une installation pilote. Présenté à un appel d'offres lancé par la C.E.E., ce projet fut l'un des deux projets solaires français retenus. Or, depuis cette étude, terminée en 1982, le contexte énergétique et économique a évolué, notamment les nouveaux tarifs proposés par E.D.F. D'autre part, et en particulier à cause de cette évolution du contexte, le développement de la filière solaire n'a pas été tel qu'on le prévoyait à l'époque. Enfin, des problèmes techniques ayant trait à la gestion des grands champs de capteurs solaires sont apparus sur les réalisations existantes.

Tout ceci nous a amené à redéfinir le projet, de manière à le rendre encore plus performant tant sur le plan technique qu'économique. Après un rappel des principales caractéristiques de l'étude initiale, nous présenterons les quelques modifications qui y ont été apportées.

\section{Le projet initial}

\subsection{Principe général}

Un stockage inter-saisonnier de chaleur dans un aquifère utilise un "doublet " de forage dont chacun fonctionne alternativement en pompage et en injection :

- en été, l'eau de la nappe est pompée à partir du forage "froid"; elle se réchauffe dans un échangeur au contact du fluide circulant dans les capteurs solaires, puis on la réinjecte dans l'aquifère par l'intermédiaire du forage " chaud";

- en hiver, le circuit est inversé; l'eau chaude stockée dans l'aquifère est pompée dans le forage "chaud" et, après avoir cédé ses calories au circuit de chauffage, elle est réinjectée dans le forage "froid". 

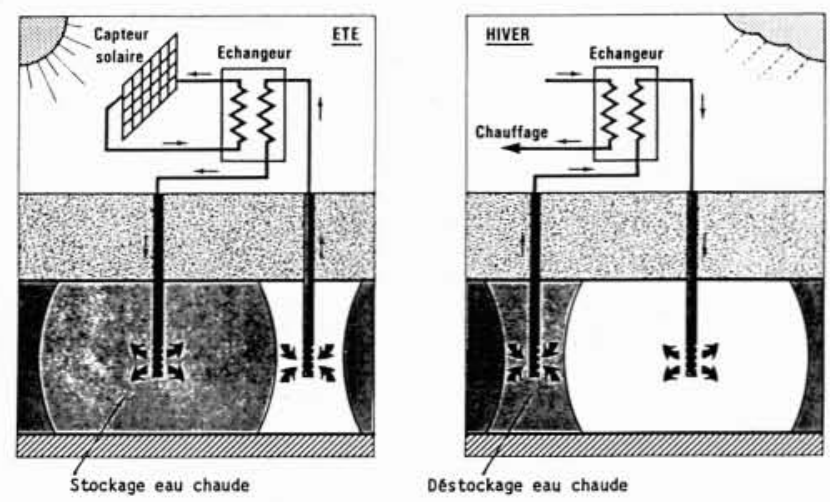

Figure 1. - Schéma de principe du stockage.

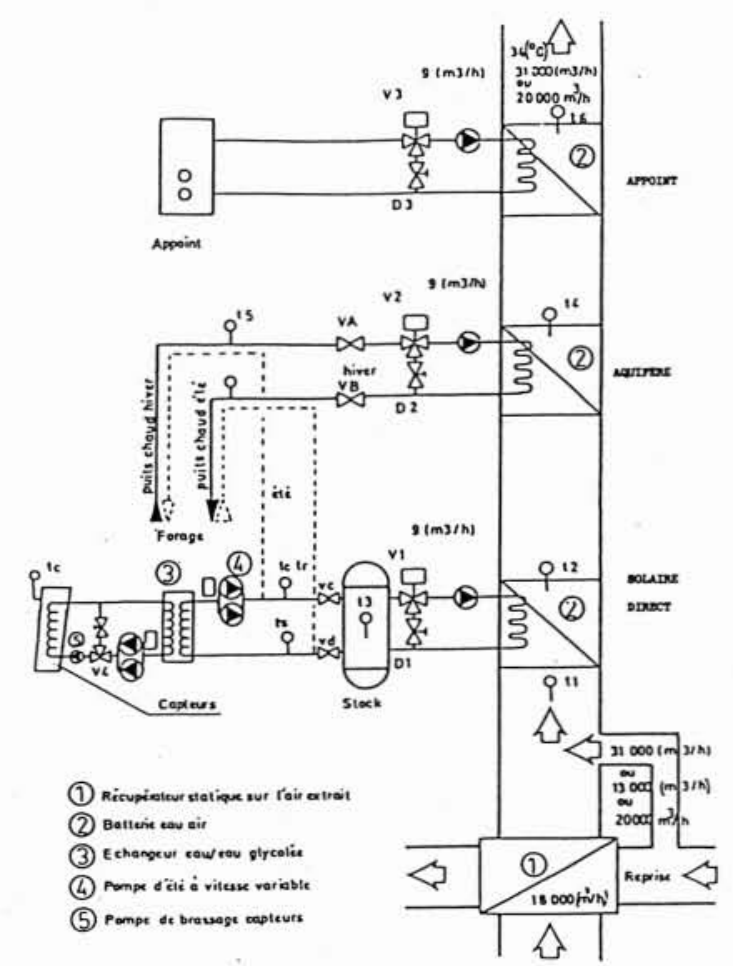

Figure 2. - Schéma de l'installation.

L'économie du stockage inter-saisonnier dépend pour l'essentiel de sa capacité d'intégration dans le système de chauffage. Celui-ci doit donc être conçu en fonction des caractéristiques du stockage et notamment utiliser un flùide à basse température afin de limiter le plus possible le recours à un appoint de chauffage conventionnel.

\subsection{Caractéristiques du projet}

Le Groupe scolaire dont il s'agit est situé sur le territoire de la Ville Nouvelle de St-Quentin-en-Yvelines. L'aquifère choisi pour le stockage est celui des Sables de Fontainebleau. Il est situé à 15 mètres de profondeur, son épaisseur est de 65 mètres et son niveau piézométrique s'établit à $25 \mathrm{~m}$ de profondeur. La perméabilité de ces sables est de 2 à $4.10^{-5} \mathrm{~m} / \mathrm{s}$; il est possible d'exploiter facilement un débit de 30 à $40 \mathrm{~m}^{3} / \mathrm{h}$.

Les besoins annuels de chauffage du groupe scolaire ont été évalués à $261,85 \mathrm{MWh}$.

Compte tenu du fort renouvellement d'air nécessaire du fait du type d'occupation des locaux, le fluide de chauffage choisi pour la distribution de la chaleur est l'air pulsé. Il est prévu, dans tous les cas, un récupérateur sur l'air extrait qui permet de récupérer $55 \mathrm{MWh}$. Le schéma de l'installation est donné sur la figure 2.

Les grandes lignes de la gestion du système sont les suivantes.

Le stockage d'été se fait par échange direct entre le fluide des capteurs et l'eau de la nappe. La température de stockage est réglée à une valeur constante de $40^{\circ} \mathrm{C}$ par modulation des débits.

En phase de chauffage, la priorité d'utilisation des trois sources de chaleur disponibles est la suivante : solaire direct, puis aquifère, puis appoint.

La régulation de la température de l'air pulsé en fonction des températures extérieures se fait par modulation des débits du réseau (solaire, aquifère, appoint).

\subsection{Performances du système - Analyse économique}

Sur ces bases, 3 configurations ont été étudiées, soit $350 \mathrm{~m}^{2}, 500 \mathrm{~m}^{2}$ et $650 \mathrm{~m}^{2}$ de capteurs, pour une année météorologique de référence et pour le capteur simple vitrage non sélectif le moins cher.

Le couplage d'un modèle de simulation des installations de surface et d'un modèle de simulation de l'aquifère a permis de suivre l'évolution du stock saisonnier au cours des 5 premières années de fonctionnement (1).

A partir de la cinquième année d'exploitation les performances sont les suivantes :

\begin{tabular}{|l|r|r|r|}
\hline \multicolumn{1}{|c|}{ Surface de capteurs } & $350 \mathrm{~m}^{2}$ & $500 \mathrm{~m}^{2}$ & $650 \mathrm{~m}^{2}$ \\
\hline Volume stocké $\left(\mathrm{m}^{3}\right)$ & 4325 & 6435 & 8438 \\
Volume déstocké $\left(\mathrm{m}^{3}\right)$ & 10880 & 11329 & 8964 \\
Température finale du stock $\left({ }^{\circ} \mathrm{C}\right)$ & 17,58 & 20,62 & 25,38 \\
Température moyenne de & 17,53 & 18,75 & 19,58 \\
réinjection (० C) & 261,85 & 261,85 & 261,85 \\
\hline Besoins (MWh) & 55,45 & 55,45 & 55,45 \\
Récupérateur (MWh) & $(21,2 \%)$ & $(21,2 \%)$ & $(21,2 \%)$ \\
Solaire (MWh) & 51,53 & 64,05 & 73,62 \\
& $(19,7 \%)$ & $(24,5 \%)$ & $(28,1 \%)$ \\
Nappe (MWh) & 88,56 & 111,99 & 117,85 \\
& $(33,8 \%)$ & $(42,8 \%)$ & $(45 \%)$ \\
Appoint (MWh) & 66,32 & 30,37 & 14,93 \\
& $(25,3 \%)$ & $(11,6 \%)$ & $(5,7 \%)$ \\
\hline Auxiliaires (MWh) & 31,8 & 32,5 & 33,9 \\
\hline
\end{tabular}

(1) Ces 5 années correspondent au temps nécessaire pour que le régime permanent soit atteint. 
Ces résultats mettent en valeur l'intérêt énergétique du système proposé, il permet notamment d'obtenir un très bon rendement de stockage (entre $75 \%$ et $53 \%$ en fonction de la surface de capteurs). De ce fait la productivité des capteurs est 2,6 à 2,7 fois supérieure à celle que l'on obtiendrait sans utiliser le stockage.

Les huit configurations concurrentes suivantes ont été comparées sur le plan économique par la méthode du coût global actualisé :

- solaire + stockage avec appoint gaz - surface de capteurs de $350 \mathrm{~m}^{2}$ (solution 1a), $500 \mathrm{~m}^{2}$ (solution $\mathrm{lb}$ ), $650 \mathrm{~m}^{2}$ (solution $1 \mathrm{c}$ ),

- solaire - stockage avec appoint électrique - surface de capteurs de $350 \mathrm{~m}^{2}$ (solution 2a), $500 \mathrm{~m}^{2}$ (solution $2 \mathrm{~b}$ ), $650 \mathrm{~m}^{2}$ (solution $2 \mathrm{c}$ ),

- classique tout électrique (solution 3),

- classique tout gaz (solution 4).

Dans le contexte économique de l'époque, les calculs faisaient apparaitre un net avantage à la solution tout gaz. Parmi les solutions solaires, les calculs du coût de la tep économisée font apparaitre la solution solaire $\left(500 \mathrm{~m}^{2}\right)+$ appoint électrique (solution $2 \mathrm{~b}$ ) comme la plus intéressante : son temps de retour était de 22 ans pour un surcoût d'investissement de l'ordre de $3 \mathrm{MF}$. C'est cette configuration qui a été présentée à la C.E.E., en vue de la réalisation d'un projet de démonstration.

\section{Le projet tel qu'il va être réalisé}

L'école retenue pour le projet sera construite sur la commune de Trappes, dans le quartier de la Plaine de Neauphle; elle devra être achevée pour la rentrée scolaire de septembre 1986.

\subsection{Caractéristiques techniques}

Le stock sera constitué en été.

Des capteurs solaires "froids", fonctionneront en source froide d'une pompe à chaleur (PAC); celle-ci cédera les calories à l'eau de la nappe, qui sera réinjectée à $50{ }^{\circ} \mathrm{C}$. La présence de la PAC permet d'assurer l'injection dans la nappe à température constante, ce qui garantit un meilleur rendement.

En hiver, l'eau est pompée au puits chaud, et cède ses calories au circuit d'air. Le stock est dimensionné pour pouvoir couvrir tous les besoins en année moyenne, mais si la température de la nappe devient trop faible, l'eau de la nappe est utilisée en source froide de la PAC (voir schéma).

Pour remplir ces conditions, il est nécessaire de stocker un volume de l'ordre de $14300 \mathrm{~m}^{3}$ à $50^{\circ} \mathrm{C}$. Le dimensionnement suivant permettra de constituer le stock :

- 2 PAC d'une puissance unitaire de $86 \mathrm{~kW}$ thermiques, - $275 \mathrm{~m}^{2}$ de capteurs "froids".

L'aquifère choisi est le même que précédemment.

Du point de vue économique, la modification du type et de la surface de capteurs nécessaires entraîne une réduction des surcoûts : ceux-ci sont d'environ 1,8 MF. Le temps de retour de l'opération, hors ingénierie, est ramené à environ 12,5 ans (2). Le financement global de l'opération est assuré de la manière suivante :
- $40 \%$ à la charge du Syndicat Communautaire d'Agglomération Nouvelle de St-Quentin-en-Yvelines, maitre de l'ouvrage.

- $30 \%$ à la charge de l'A.F.M.E.,

- $30 \%$ à la charge de la Communauté Économique Européenne.

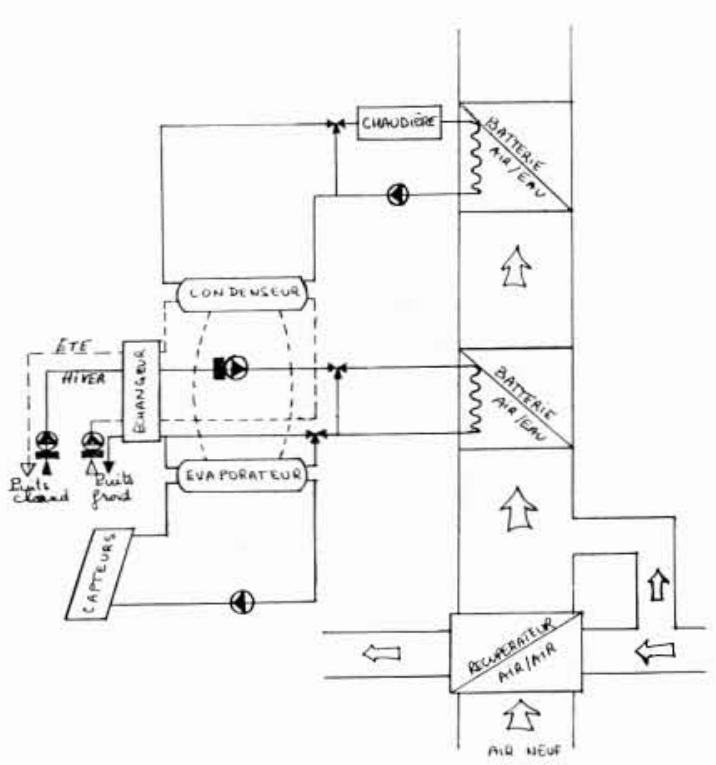

Figure 3

(2) Les résultats indiqués ci-dessus ont été tirés des données de l'étude, sans avoir mis en cuvre les modéles de simulation. L'avant-projet détaillé est en cours de préparation.

\section{Discussion}

Président : M. B. Dessus

Les rendements de restitution sont obtenus en calculant le quotient de l'énergie récupérée par l'énergie injectée (question de M. DESPOIS).

Le stock est constitué à une température de $50 \circ \mathrm{C}$ environ. Afin de pouvoir couvrir la totalité des besoins de chauffage, et compte tenu du type de chauffage, qui impose une température résiduelle de 35 à $40{ }^{\circ} \mathrm{C}$. on doit injecter plus d'énergie que ce qui est nécessaire. Les rendements de restitution sont alors inférieurs à loptimum.

En ce qui concerne le captage de la nappe des sables de Fontainebleau, il est difficile aux forts débits, mais dans le projet présenté, le débit sera au maximum de $15 \mathrm{~m}^{3} / \mathrm{h}$ (question de $M$. GUEDE). En soignant l'installation, la longévité des forages devrait être très bonne malgré la succession des pompages et des injections. 


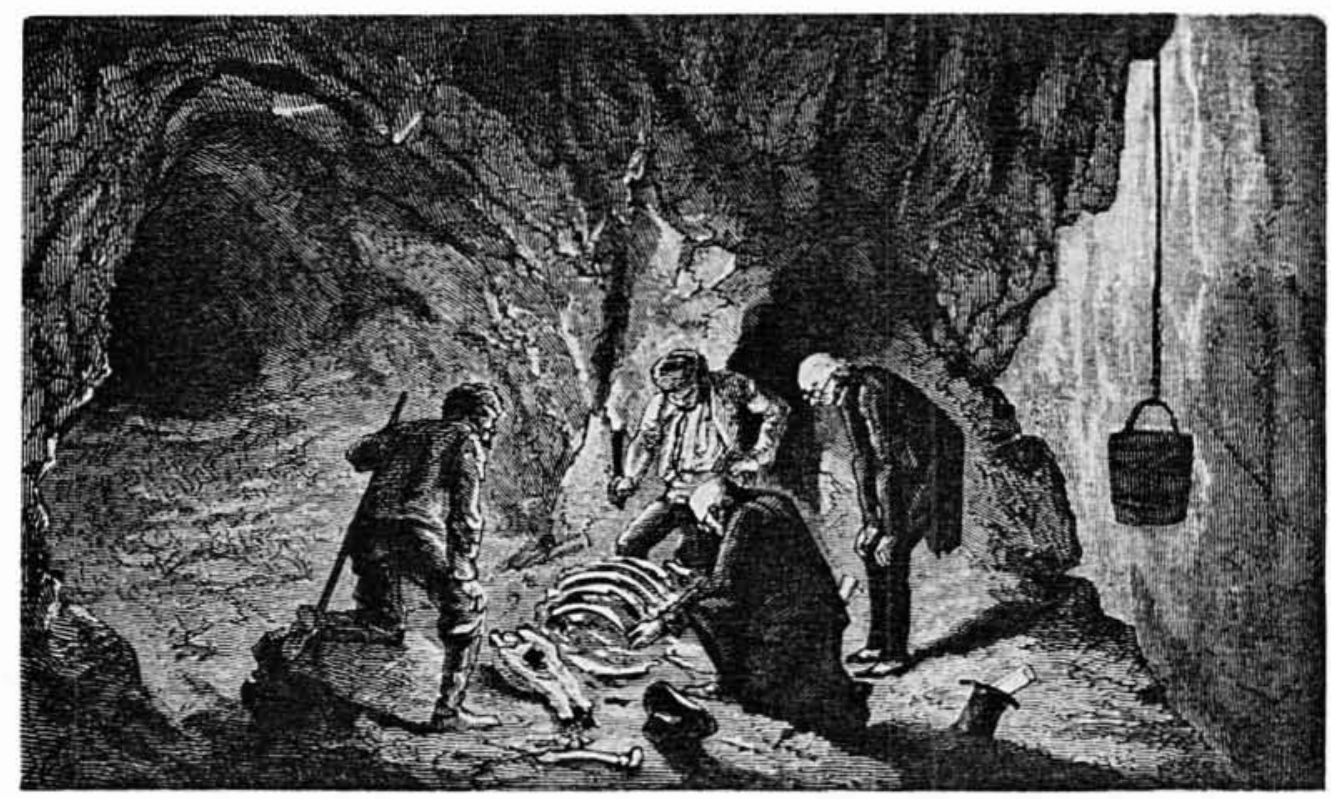

